\title{
Some of My Favorite "Lesser Known” Problems
}

\author{
Moshe Rosenfeld * \\ Computing and Software Systems Program, \\ University of Washington, Tacoma, WA 98402, USA
}

Received 13 August 2007, accepted 4 November 2008, published online 10 November 2008

\begin{abstract}
Some problems become famous and attract attention while others collect dust on the shelf. Large conferences are a great breeding ground for popularizing both old and new problems. In this note we present some off the shelf problems.
\end{abstract}

Keywords: Graph coloring, partitions.

Math. Subj. Class.: 05C15, 05B45, 05A17

\section{Introduction}

What makes problems popular? Some of the factors that add to the popularity of a problem include: its author, attractiveness, its simplicity and money. Money usually arrives after a problem becomes popular. A case in point are the millennium problems. Even though many researchers are attracted to the problems just because of their challenge, offering a $\$ 1,000,000.00$ prize money for their solution extends their popularity to the population at large. A key role is also played by the person publicizing the problem. Probably the strongest example is David Hilbert. On August 8, 1900, David Hilbert delivered his famous lecture about open mathematical problems at the second International Congress of Mathematicians in Paris. His list of twenty three problems had a profound and lasting influence on mathematical research. Another example is Paul Erdős who delivered numerous talks and published many papers about open problems, occasionally offering prizes for their solutions and frequently repeating the same problems, in different venues giving them more exposure.

Consider for example the famous unit-distance problem. This problem asks for the smallest number of colors needed to color the points of the plane $R^{2}$ so that points unit distance apart have distinct colors. This problem was attributed to at least five different mathematicians (in a variety of combinations): Edward Nelson, Hugo Hadwiger, Paul Erdős, Martin

\footnotetext{
* The author would like to acknowledge support by the Institute of Technology at UWT.

E-mail address: moishe@u.washington.edu (Moshe Rosenfeld)
} 
Gardner and Leo Moser (was this a factor that helped make it popular?) It is now known as the Hadwiger-Nelson problem [4]. The problem was actually formulated by Edward Nelson in 1950 when he was a graduate student at the University of Chicago. Nelson called it the alternative four-color problem as it dealt with the plane and four colors. He proved that at least four colors are needed. John Isbell, upon learning about the problem from Nelson, proved in 1951 that the unit-distance graph is 7-colorable. This problem might be a typical candidate for a famous problem. It is simple enough for a high-school student to understand, it is easy to describe and looks deceptively easy to solve. After all, all one needs is to construct a finite set of points in the plane that requires more than 4 colors or prove that any finite set of points is 4-colorable. What can be simpler than this? As a matter of fact, while hundreds of papers dedicated to variations of this problem have been published, no progress has been made on the actual chromatic number of the unit-distance graph since its inception. This has probably added to its popularity. For an interesting account of the history of this problem see [10].

In this note I present four lesser known problems I tried to solve.

1. The odd-distance graph.

2. The Salmon life-cycle problem.

3. Partitioning the unit square into rectangles.

4. A numerical matching problem.

In section 2 we discuss a close relative of the unit distance graph: the odd-distance graph. In section 3 we introduce a graphically attractive new problem. In section 4 we discuss an elementary problem dealing with partitioning the unit square into rectangles. In section 5 we conclude with an elementary problem in number theory. My experience is that repeated exposure of problems in conferences and workshops eventually leads to progress, be it a complete solution or related venues of research. I hope this will also happen here.

\section{The odd-distance graph}

This problem was motivated by problem B-5 that appeared in the 1993 Putnam competition: Show there do not exist four points in the Euclidean plane such that the pairwise distances between the points are all odd integers. For a short proof see [8]

In other words, the graph whose vertices are the points in the plane $R^{2}$ and edges between pairs of points at an odd distance apart, does not contain $K_{4}$ as a subgraph. We call this graph the odd-distance plane graph. In 1994, at the $25^{\text {th }}$ Southeastern Conference on Combinatorics, Graph Theory and Computing in Boca Raton, I asked Paul Erdôs whether the odd-distance plane graph can be colored by a finite number of colors. Erdôs included this question in his open problems talk at the conference and added his own favorite twist: what is the maximum number of odd distances among $n$ points in the plane, [5]. This is the same question he raised in 1946 on the unit-distance graph [6]: what is the maximum number of unit distances possible among $n$ points in the plane (another famous still open problem).

This was the first exposure of the odd-distance plane graph problem. It instantly attracted attention. The first related result was a paper by L. Piepmeyer [7]. He showed that the Turán graph $K_{n, n, n}$ is a subgraph of the odd-distance graph thus proving that $3 n^{2}$ odd integral distances can occur among $3 n$ points in the plane. By Turán's theorem, this is best possible. 
Clearly, the unit-distance plane graph is a subgraph of the odd distance graph, thus the chromatic number of the odd-distance plane graph is at least 4 . Only recently, we managed to prove that the chromatic number of the odd-distance plane graph is at least 5 [3]. In this paper we also proved that this graph is $\aleph_{0}$-choosable while the odd-distance graph in $R^{3}$ is not countably choosable, a marked difference from the unit-distance graph in $R^{3}$. We conclude this section with a list of problems.

- Is the chromatic number of the odd-distance graph finite?

- Construct triangle-free graphs which are not subgraphs of the odd-distance plane graph.

I believe that such graphs exist. For the unit-distance plane graph they are easy to construct. Any graph with arbitrarily high girth and chromatic number greater than 7 is not a subgraph of the unit-distance graph. The only graphs that are not subgraphs of the odd distance graph which I know are graphs that contain $K_{4}$ as a subgraph.

- Obtain lower bounds for the chromatic number of the $R^{3}$ odd-distance graph.

The best known lower bound for the $R^{3}$ unit-distance graph is 6 . I am sure that this bound can be easily improved.

- Is $K_{n, n, n, n}$ a subgraph of the $R^{3}$ odd-distance graph?

If true then this will give the maximum number of odd integral distances among points in $R^{3}$.

\section{The "Salmon" problem}

This problem was motivated by an old cute well-known problem:

You have a stick 100 inches long. You randomly distribute 101 "bugs" on it. The bugs move at a speed of $1 \mathrm{inch} / \mathrm{min}$. Once they start moving they don't change direction unless they collide with another bug. When they reach the end of the stick they "fall off" (die). All bugs start moving at time 0 . Initial direction for each bug is randomly chosen. Will all bugs eventually die and if so when?

The solution is very nice in its simplicity. All bugs will die after no more than $100 \mathrm{~min}-$ utes. To see why, just color all bugs that move to the left red and those that move to the right blue. When two bugs collide, just change their colors. This means that all colors never change their directions and move at a constant speed of $1 \mathrm{inch} / \mathrm{min}$ towards their eventual demise after at most 100 minutes.

We propose a variation on this problem. The life cycle of the salmon starts at the river where they are hatched, continues in a long loop (one to three years) in the ocean, followed by a return to their birth place spawn and die. Consider now a cycle with $n$ randomly distributed points "salmon fries" on it. Each point moves in a constant speed either clockwise or counter clockwise (direction randomly assigned). When two fries collide they reverse direction. When a fry returns to its origin, it dies. If there is a collision at the point of origin, 
then the death takes precedence and the other fry continues in the same direction.

My student Tim Minalia wrote a Java program to simulate this problem. The problem randomly placed fries, each assigned a random direction, and started the motion. While in almost all cases all fries died, in some cases it seemed as if two or four fries were destined to live forever. For instance, if fry A started at 90 degrees, fry B started at 270 degrees and after some collisions all other fries died while $\mathrm{A}$ is in location 181 degrees and $\mathrm{B}$ is in location 179 degrees and both are moving in opposite directions then it is easy to see that none of them will ever reach its origin. Together with Ján Manǔch we started a closer scrutiny of this problem. We found the following two sample runs interesting (see Table 1):

\begin{tabular}{|c|ccccc|}
\hline Time & S1 & S2 & S3 & S4 & S5 \\
\hline 0 & -112 & -114 & -16 & -70 & +74 \\
\hline 19 & +93 & -95 & -125 & -51 & -93 \\
\hline 20 & -94 & +94 & -124 & -50 & -92 \\
\hline 35 & -79 & -109 & +109 & -35 & -77 \\
\hline 38 & -76 & -106 & +112 & -32 & die \\
\hline 62 & -52 & -82 & -8 & +8 & \\
\hline 84 & +30 & -60 & -114 & -30 & \\
\hline 99 & -45 & +45 & -99 & -15 & \\
\hline 126 & -18 & -72 & +72 & -116 & \\
\hline 148 & -124 & -50 & -94 & +94 & \\
\hline 160 & die & -38 & -82 & +106 & \\
\hline 190 & & +8 & -52 & -8 & \\
\hline 212 & & -30 & +30 & -114 & \\
\hline 254 & & -116 & -72 & +72 & \\
\hline 256 & & die & -70 & +74 & \\
\hline 310 & & & die & +0 & \\
\hline 380 & & & die & \\
\hline
\end{tabular}

Table 1: The life cycle of five salmon fries.

At time 0 we mark the initial location of each fry and its assigned direction: + means clockwise and - counter clockwise. The first column records the elapsed time. Each line records the first collision. Note that a full cycle takes 128 seconds. All arithmetic is done mod 128. It took more than 3 full cycles for all fries to die. It does not look obvious how we could predict the first death, the death sequence and the final death.

A second example produced a more intriguing situation. We started again with five fries located as indicated in Table 2.

In this example four fries will move forever. We skipped all intermediate collisions (the reader could verify them). It is easy to see that none of them will ever reach its origin. Indeed as the program continues to run it will produce exactly the same configuration later. These initial observations led us to ask the following questions:

- Given as input $n$ fries (locations on the cycle) and directions. Is there an efficient algorithm to decide whether they will all die? 


\begin{tabular}{|c|ccccc|}
\hline Time & S1 & S2 & S3 & S4 & S5 \\
\hline 0 & +120 & +92 & -55 & -41 & -51 \\
\hline 110 & die & +102 & -100 & -0 & +74 \\
\hline 123 & & -115 & +87 & +115 & -87 \\
\hline
\end{tabular}

Table 2: Reaching eternity

- for an integer $n$ determine the maximum number of collisions for all inputs that die.

- For a given integer $n$, determine what is the longest time a colony can live before they all die?

In other words, is there a threshold beyond which if some fries are still alive then they'll never die.

- Is it possible for an odd number of fries to survive forever?

In our examples, we observed that an even number of fries survived.

\section{Partitioning the unit square}

How should one partition the unit square into $n$ rectangles with sides parallel to the sides of the square so that the largest perimeter among the rectangles will be minimized? This is an elementary problem that is easily understood by high school students. The problem actually originated as a data allocation problem in parallel computing and in a scheduling problem as reported in [2]. When $n=k^{2}$ the solution is obvious. It is quite surprising that a simple problem like this has attracted very little attention. When all rectangles are assumed to have the same area, the solution is simple. It was shown in [9] that if $k^{2}<n<(k+1)^{2}$ then there must be rectangles whose longer side is at least $\frac{1}{k}$ and also rectangles whose shorter side is at most $\frac{1}{k+1}$. It is then easy to partition the square into $m_{1}$ rows of rectangles of size $\frac{1}{k} \times \frac{k}{n}$ and $m_{2}$ rows of $\frac{1}{k+1} \times \frac{k+1}{n}$ (where $n=m_{1} \times k+m_{2} \times(k+1)$ ) which yield the minimal largest perimeter. Furthermore, a direct consequence of this construction is that in general, the largest perimeter is not obtained when all rectangles have the same area. The general case seem to be much more difficult. Anderson conjectured that the optimal partition is obtained when all rectangles have the same perimeter. In 1986 Noga Alon and Daniel Kleitman proved that when $n=k(k+1)$ the partition of the square into $n$ rectangles of size $\frac{1}{k} \times \frac{1}{k+1}$ minimizes the largest perimeter [1]. More recently, they also proved that partitioning the square into $k-1$ rows of $k$ identical rectangles and an additional row of $k+s, s= \pm 1$ rectangles such that all rectangles have the same area also yields the optimal solution for $n=k^{2} \pm 1$. No other cases have been resolved.

\section{A numerical matching problem}

Given a partition of the integers $1,2, \ldots, 2 n$ into two equal sets $A=\left\{a_{1}, \ldots a_{n}\right\}$ and $B=\left\{b_{1}, \ldots b_{n}\right\}$. Is it always possible to match the numbers in A with the numbers in $\mathrm{B}$ such that $\left\{ \pm\left(a_{i}-b_{i}\right)(\bmod 2 n+1)\right\}=\{1,2, \ldots, 2 n\}$ ?

This problem was a natural extension of a theorem that proves that the quadratic residues 
in a finite field $G F(q)$ can be matched with the non-quadratic residues when $q \geq 7$. For example Table 3 shows a matching of the quadratic residues in GF(41) with the non-residues.

\begin{tabular}{|cccccccccc|}
\hline 1 & 8 & 23 & 20 & 37 & 9 & 31 & 2 & 16 & 5 \\
\hline 7 & 15 & 38 & 17 & 13 & 22 & 12 & 14 & 30 & 35 \\
\hline \hline 40 & 33 & 18 & 21 & 4 & 32 & 10 & 39 & 25 & 36 \\
\hline 34 & 26 & 3 & 24 & 28 & 19 & 29 & 27 & 11 & 6 \\
\hline
\end{tabular}

Table 3: Quadratic residues matching in GF(41).

For $3 \leq n \leq 9$ all partitions of $1,2, \ldots, 2 n$ where shown to be matchable with two essentially distinct exceptions of order 7 . See tables 4 and 5

\begin{tabular}{|ccccccc|}
\hline 1 & 4 & 5 & 6 & 9 & 11 & 14 \\
\hline 2 & 3 & 7 & 8 & 10 & 12 & 13 \\
\hline
\end{tabular}

Table 4: A non-matchable partition of order 7.

\begin{tabular}{|lllcccc|}
\hline 1 & 3 & 4 & 7 & 10 & 12 & 13 \\
\hline 2 & 5 & 6 & 8 & 9 & 11 & 14 \\
\hline
\end{tabular}

Table 5: A non-matchable partition of order 7.

In 2003 I presented this question in the Czech-Slovak conference in Javorna. During the conference Roman Sotak produced an example of non matchable particular partitions $\forall n=5 k+2 k \geq 1$. I believe that the non-matchable partitions are very rare. It would be nice to find them all or at least more examples. In particular we ask whether for almost all $n$ all partitions are matchable.

\section{Acknowledgements}

The author would like to thank the organizers of the $6^{\text {th }}$ Slovenian International Conference of Graph Theory Bled'07 for a great, inspiring conference.

The author would also like to thank the referees for their meticulous review and constructive suggestions.

\section{References}

[1] N. Alon and D. Kleitman, Covering a square by small perimeter rectangles, Disc. Computational Geometry 1 (1986), 1-7.

[2] N. Alon and D. Kleitman, Partitioning a rectangle into small perimeter rectangles, Discrete Math. 103 (1992), 111-119.

[3] H. Ardal, J. Manǔch, M. Rosenfeld, S. Shelah and L. Stacho, The Odd-distance Plane Graph, Submitted. 
[4] P. Brass, W. Moser and J. Pach, Research Problems in Discrete Geometry, Springer, New York, 2005, p. 234.

[5] P. Erdôs, Twenty five years of questions and answers, $25^{\text {th }}$ Southeastern International Conference on Combinatorics, Graph Theory and Computing, Boca Raton, Florida 1994.

[6] P. Erdős, On sets of distances of $n$ points, Amer. Math. Monthly 53 (1946), 248-250.

[7] L. Piepmeyer, The Maximum Number of Odd Integral Distances Between Points in the Plane, Discrete \& Computational Geometry 16 (1996), 113-115.

[8] M. Rosenfeld, Odd integral distances among points in the plane, Geombinatorics 5 (1996), 156159.

[9] M. Rosenfeld, Partitioning a square into rectangles, Congr. Numer. 54 (1986), 101-104.

[10] A. Soifer, Chromatic number of the plane \& its relatives Part I: the problem \& its history, Geombinatorics 12 (2003), 131-148. 